\title{
Risk Evaluation of Bogie System Based on Extension Theory and Entropy Weight Method
}

\author{
Yanping Du, ${ }^{1,2}$ Yuan Zhang, ${ }^{1,2}$ Xiaogang Zhao, ${ }^{3}$ and Xiaohui Wang ${ }^{1}$ \\ ${ }^{1}$ School of Mechanical Engineering, Beijing Institute of Graphic Communication, Beijing 102600, China \\ ${ }^{2}$ State Key Laboratory of Rail Traffic Control and Safety, Beijing Jiaotong University, Beijing 100044, China \\ ${ }^{3}$ School of Electromechanical and Vehicle Engineering, Beijing University of Civil Engineering and Architecture, Beijing 100044, China
}

Correspondence should be addressed to Yuan Zhang; zhangyuan111@gmail.com

Received 29 September 2014; Accepted 26 November 2014; Published 10 December 2014

Academic Editor: Yongjun Shen

Copyright (C) 2014 Yanping Du et al. This is an open access article distributed under the Creative Commons Attribution License, which permits unrestricted use, distribution, and reproduction in any medium, provided the original work is properly cited.

\begin{abstract}
A bogie system is the key equipment of railway vehicles. Rigorous practical evaluation of bogies is still a challenge. Presently, there is overreliance on part-specific experiments in practice. In the present work, a risk evaluation index system of a bogie system has been established based on the inspection data and experts' evaluation. Then, considering quantitative and qualitative aspects, the risk state of a bogie system has been evaluated using an extension theory and an entropy weight method. Finally, the method has been used to assess the bogie system of four different samples. Results show that this method can assess the risk state of a bogie system exactly.
\end{abstract}

\section{Introduction}

With the rapid development of China's public rail transport, the train being the most important tool and the direct carrier of passengers' and goods in rail transport, its safety is receiving more and more attention. The safe and efficient operation of a train depends directly on the operation state of its key systems or components. The bogie system directly bears the weight of the carriage and its load and has the role of load bearing, moving, traction, and guiding. It can also mitigate the impact between the wheels and rail, reducing vehicle vibration and ensuring the safe and smooth operation of the train. It can effectively brake, to ensure safe stopping of the train. It is one of the most important parts to support the safe operation of the train. Accurate analysis and evaluation of the operation state of the train bogie system can effectively prevent accidents and improve the ability of active prevention. This can provide support for the train operation management departments in train repair and maintenance, fault prevention, and scheduling decisions.

At present, research on the bogie system of trains has been focused on two aspects. One is the fault diagnosis and state monitoring of the parts involved, for example, the fault diagnosis of wheel sets $[1,2]$, research on online monitoring systems [3], fault diagnosis [4, 5], and evaluation [6] of bearings, and research on fault diagnosis methods for elastic suspension devices [7-9]. The other approach is a subjective evaluation method that carries out safety evaluation of the bogie system based on expert experience [10].

The above research has had effective results, but on the whole research on the risk analysis and evaluation of the bogie system is still lacking. There may also be overreliance on subjective factors in qualitative assessments. This paper aims to establish a risk evaluation system for the bogie system based on monitored data of the state of key elements of the bogie system and expert experience and uses extension theory and entropy weight method to carry out an evaluation of the bogie system.

\section{Risk Evaluation System of Bogie Systems}

The bogie system consists of the crankcase, wheel set, traction drive device, foundation brake rigging, elastic suspension device, frame, grounding device, and so forth. This paper aims to establish a risk evaluation method for the bogie 
system using the above mentioned key elements as basic factors.

2.1. Evaluation Index for Roller Bearings. At present, fault diagnosis technology for roller bearings based on signal processing of vibration acceleration is mature. It is necessary to accurately detect the status change of roller bearings when its risk state is being evaluated. Therefore, using the vibration signal of roller bearings, calculations can accurately reflect the time domain parameters of the trend variation of roller bearings which can be used as a risk evaluation index. Time domain parameter is a simple method for the detection and diagnosis of early faults of rolling bearings. It includes effective value, peak value, peak factor, kurtosis, pulse factor, margin factor, waveform factor, and so forth [11-14]. Therefore, this paper uses the effective value, peak factor, and kurtosis as risk evaluation indices for bearings. For method of calculation refer to [14].

2.2. Evaluation Index for Wheel Sets. Wheel diameter is the diameter of the wheel tread. Friction of wheel treads results in a reduction of wheel diameter, affecting the dynamics of the railcar. The wheel rim is an important part that ensures that the train moves along the track and prevents derailment. A reasonable wheel rim width will ensure the train proceeds safely through the turns and prevents collision of the wheel rim and bolt connections of the rail. As to wheel diameter, to ensure the safety of the train, there are requirements relating to the diameter difference of two wheels in the same shaft and diameter difference of four wheels in the same bogie. Taking the metro vehicle as an example, the range value of wheel diameter is generally $840-770 \mathrm{~mm}$ [15-17]. The range value of wheel rim width is $32-26 \mathrm{~mm}$ [15-17]. The diameter difference between the left wheel and right wheel in the same shaft should be less than $2 \mathrm{~mm}$ [15-17]. The diameter difference between the four wheels in the same bogie should be less than $4 \mathrm{~mm}$ [15-17]. Therefore, to evaluate the impact of wheel set risk on the whole bogie system, this paper uses wheel diameter, wheel rim width, difference in diameter between coaxial wheels, and difference in diameter between co-bogie wheels as risk evaluation indices for wheel sets.

2.3. Evaluation Index for Traction Drive Devices. Traction motor is often referred to as the "heart" of the train. It is an important part in the normal operation of the train [18]. At present, techniques for fault diagnosis of traction motors can be based on vibration signal, temperature monitoring, electrical current, and so forth [19]. This paper mainly studies the monitoring of hidden risk states of traction motors. It is hoped that the monitoring index can be obtained rapidly, so as to carry out the risk assessment of the bogie system. Therefore, this paper chooses the temperature of the traction motor as the risk evaluation index.

2.4. Evaluation Index for Foundation Brake Rigging. Brake shoe of the foundation brake is the most commonly used method in train braking. In the maintenance of metro vehicles, the thickness of the brake shoe must be measured and recorded. Attention is needed when the thickness of the brake shoe is $17.5-18 \mathrm{~mm}$. The brake shoe needs to be changed when its thickness is not more than $15 \mathrm{~mm}$. Brake shoes of different thicknesses have different impact on the operation of the train. It is known from the statistical records of accidents of a local railway network that $56 \%$ of braking system faults can be attributed to brake shoe faults.

2.5. Other Evaluation Indices. Few specialized sensors are installed on trains for monitoring the state of elastic suspension device, bogie frame, and grounding device. Therefore, for these kinds of devices, risk evaluation is carried out by an expert scoring method. Based on expert experience, we chose risk parameters of the elastic suspension device, bogie frame, and grounding device and fit them into the risk evaluation index system of the bogie system.

2.6. Risk Evaluation System for Bogie Systems. According to the above analysis and principles, this paper establishes risk evaluation index method for the bogie system, which is shown in the flow chart of Figure 1.

\section{Extension Theory and Entropy Weight Method}

The risk evaluation of the bogie system is a complex and contradictory problem. When carrying out the evaluation, it is necessary to consider quantitative information, such as monitoring and maintenance records, and qualitative knowledge, such as expert experience. Extension theory has been developed in recent years for the study of contradictions in the real world. It mainly deals with contradictory problems from two aspects: the qualitative and the quantitative [20]. The entropy weight method is an objective weighting method [21]. According to the degree of variation of each index, the information entropy can be calculated. The weight of an index is determined by its entropy. The larger the entropy, the smaller the usefulness of the index and, therefore, the smaller the weight. On the contrary, the smaller the entropy, the greater the weight. This paper combines extension theory with the entropy weight method and applies it to the risk evaluation of the bogie system. The steps for risk evaluation based on extension theory and entropy weight method are briefly described below.

(1) Classification of Risk Level. If the risk of the evaluation object is classified into $m$ levels, the set of comments used in the evaluation is

$$
\begin{aligned}
M & =\left\{M_{1}, M_{2}, \ldots, M_{4}\right\} \\
& =\{\text { hazard level } A, \text { hazard level } B, \ldots, \text { hazard level } m\}
\end{aligned}
$$

(2) Determination of the Standard Domain and the Extensional Domain. Standard domain is the range of values of each 


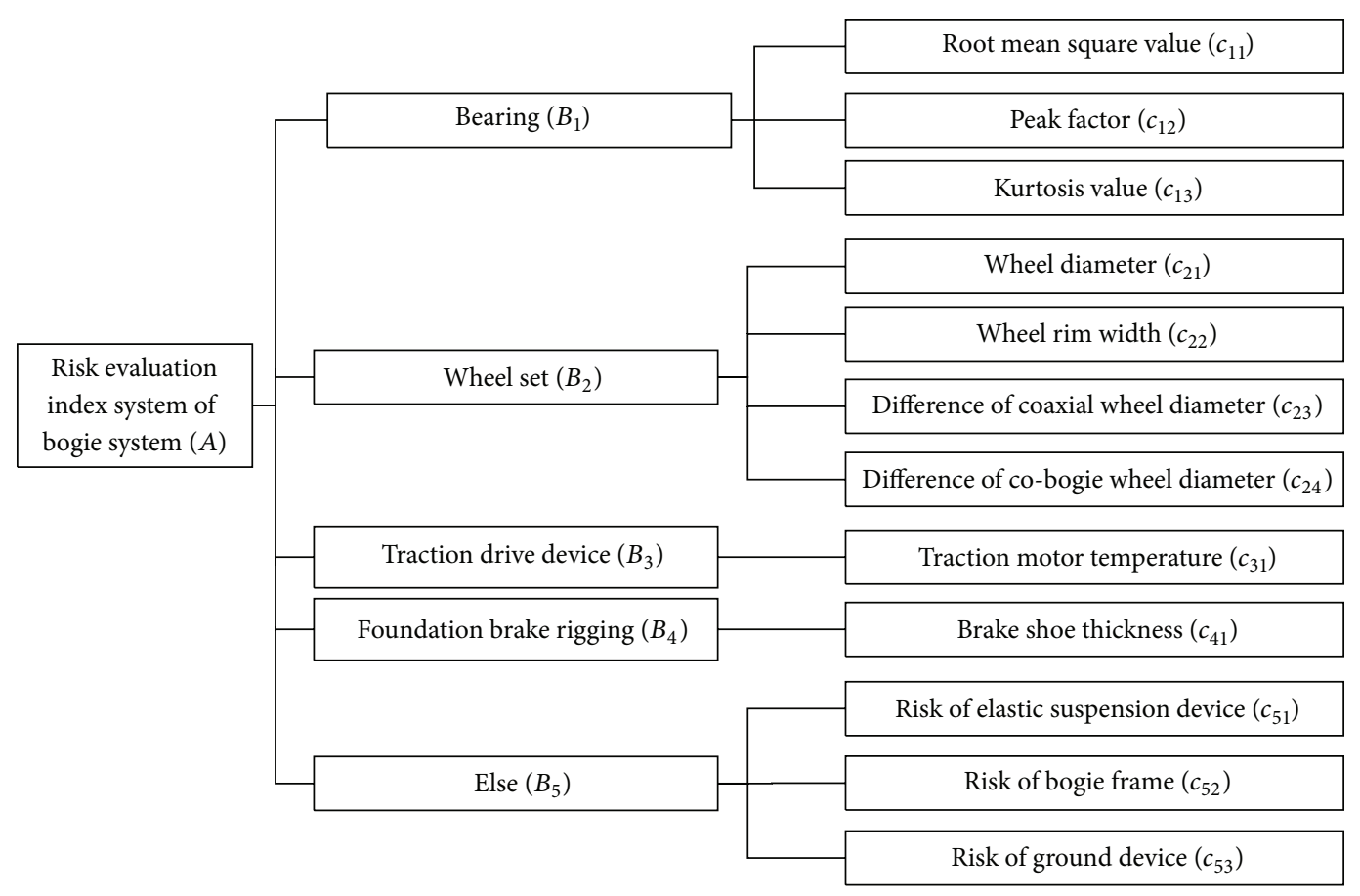

FIGURE 1: Risk evaluation index system of bogie system.

index corresponding to each hazard level. Standard matterelement can be established based on the evaluation criteria:

$$
\begin{aligned}
R_{0} & =\left[\begin{array}{ccccc}
M & M_{01} & M_{02} & \cdots & M_{0 m} \\
c & v_{01} & v_{02} & \cdots & v_{0 n}
\end{array}\right] \\
& =\left[\begin{array}{ccccc}
M & M_{01} & M_{02} & \cdots & M_{0 m} \\
c_{1} & \left\langle a_{11}, b_{11}\right\rangle & \left\langle a_{12}, b_{12}\right\rangle & \cdots & \left\langle a_{1 m}, b_{1 m}\right\rangle \\
c_{2} & \left\langle a_{21}, b_{21}\right\rangle & \left\langle a_{22}, b_{22}\right\rangle & \cdots & \left\langle a_{2 m}, b_{2 m}\right\rangle \\
\vdots & \vdots & \vdots & \vdots & \\
c_{n} & \left\langle a_{n 1}, b_{n 1}\right\rangle & \left\langle a_{n 2}, b_{n 2}\right\rangle & \cdots & \left\langle a_{n m}, b_{n m}\right\rangle
\end{array}\right] .
\end{aligned}
$$

In the formula, $R_{0}$ is the same matter-element body of the same matter-elements $R_{1}, R_{2}, \ldots, R_{n}$, and it represents all the status of the evaluation categories; $n$ is the number of hazard indexes according to the concept of matter-element theory; $c_{i}$ is the $i$ th risk evaluation index of the object to be evaluated; $v_{0 m}$ is the range of values of $M_{0 m}$ relating to characteristic $c_{i} ; M_{0 m}$ is the $m$ th evaluation grade of the object to be evaluated; the $\left\langle a_{n m}, b_{n m}\right\rangle$ that it corresponds to is the standard domain.

Extensional domain is the range of all possible values of an object in its entire life cycle. The extensional domain of the risk level to be evaluated is

$$
\begin{aligned}
R_{P}= & \left(M_{P}, c, v_{P}\right) \\
= & {\left[\begin{array}{ccc}
M_{P} & c_{1} & v_{P 1} \\
& c_{2} & v_{P 2} \\
& \vdots & \vdots \\
& c_{n} & v_{P n}
\end{array}\right]=\left[\begin{array}{ccc}
M_{P} & c_{1} & \left\langle a_{P 1}, b_{P 1}\right\rangle \\
& c_{2} & \left\langle a_{P 2}, b_{P 2}\right\rangle \\
& \vdots & \vdots \\
& c_{n} & \left\langle a_{P n}, b_{P n}\right\rangle
\end{array}\right] . }
\end{aligned}
$$

In the formula, $M_{p}$ is all the elements of the comment set; $v_{p n}$ is the range of values of $c_{n}$; the $\left\langle a_{P n}, b_{P n}\right\rangle$ that it corresponds to is the extensional domain.

(3) Determination of the Matter-Element to Be Evaluated. The matter-element under evaluation refers to the index value of the evaluation object at the time of evaluation:

$$
R=(M, c, v)=\left[\begin{array}{ccc}
M & c_{1} & v_{1} \\
& c_{2} & v_{2} \\
& \vdots & \vdots \\
& c_{n} & v_{n}
\end{array}\right] .
$$

In the formula, $R$ is the matter-element under evaluation; $M$ is the evaluation object; $v_{i}$ is the value of $c_{i}$.

(4) Establishing the Correlation Function to Calculate the Degree of Correlation. The correlation function of $i$ th index of the evaluation object in relation to the level of risk state $j$ can be calculated by the following formula:

$$
\begin{aligned}
& K_{j}\left(v_{i}\right) \\
& =\left\{\begin{array}{l}
\frac{\rho\left(v_{i}, v_{0 j i}\right)}{\rho\left(v_{i}, v_{P i}\right)-\rho\left(v_{i}, v_{0 j i}\right)} \\
v_{i} \notin v_{0 j i} \\
-\frac{\rho\left(v_{i}, v_{0 j i}\right)}{\left|v_{0 j i}\right|} \\
v_{i} \in v_{0 j i} \text { and } \rho\left(v_{i}, v_{P j}\right)=\rho\left(v_{i}, v_{0 j i}\right) .
\end{array}\right.
\end{aligned}
$$


In the formula, $K_{j}\left(v_{i}\right)$ is the degree of correlation; $\rho\left(v_{i}, v_{0 j i}\right)$, $\rho\left(v_{i}, v_{P i}\right)$ are the distances from point $v_{i}$ to the standard domain and the extensional domain, respectively. Consider

$$
\begin{gathered}
\rho\left(v_{i}, v_{0 j i}\right)=\left|v_{i}-\frac{a_{0 j i}+b_{0 j i}}{2}\right|-\frac{b_{0 j i}-a_{0 j i}}{2} \quad(i=1,2, \ldots, n), \\
\rho\left(v_{i}, v_{P i}\right)=\left|v_{i}-\frac{a_{P i}+b_{P i}}{2}\right|-\frac{b_{P i}-a_{P i}}{2} \quad(i=1,2, \ldots, n) .
\end{gathered}
$$

After the computation, the degree of correlation matrix of each index in relation to hidden risk level $K=\left(k_{i j}\right)_{n \times m}$ can be obtained.

(5) Determining the Weights for Each Index. Then, the entropy weight method is used to calculate the weight of the indices. The detailed process is as follows.

(1) If the bogie system is classified into $m$ levels when evaluated, there will be $n$ evaluation indices. Establish a judgment matrix of size $n \times m$. This is the correlation matrix $K=\left(k_{i j}\right)_{n \times m}$ solved in the previous section.

(2) After correlation matrix $s_{i j}=k_{i j} / \sum_{j=1}^{m} k_{i j}$ is normalized, matrix $S=\left(s_{i j}\right)_{n \times m}$ can be obtained.

(3) Calculate the entropy of the index evaluated using the following formula:

$E_{i}=-Q \sum_{j=1}^{m} s_{i j} \ln s_{i j} \quad(i=1,2,3, \ldots, n ; j=1,2,3, \ldots, m)$.

If $s_{i j}=0$, then specify $s_{i j} \ln s_{i j}=0$; therefore, $0 \leq E_{i} \leq$ 1 :

$$
Q=\frac{1}{\ln n}
$$

(4) Calculate degree of variation coefficient of the indices using the following formula:

$$
d_{i}=1-E_{i}
$$

(5) Calculate the entropy weight coefficient of the indices using the following formula:

$$
\omega_{i}=\frac{d_{i}}{\sum_{i=1}^{n} d_{i}}
$$

(6) Determination of Risk Level. Considering the weights of each characteristic, the comprehensive correlative degree is obtained by combining the correlation degree and the weight coefficient:

$$
K_{j}(M)=\sum_{i=1}^{n} w_{i} K_{j}\left(v_{i}\right)
$$

If $K_{j 0}(M)=\max \left\{K_{j}(M) j=1,2, \ldots, m\right\}, M$ is evaluated as level $j_{0}$.

\section{A Case of Risk Evaluation of Bogie System}

To verify the validity of the method, we chose the bogie system of experimental trains of a metro company as the object of research.

(1) Classification in Risk Evaluation. According to the state processing method of bogie systems, the risk level is classified into three grades. The seriousness of risk increases step by step as the risk level proceeds from low to high. Low risk indicates that the bogie system can still function normally; medium risk indicates that the bogie system can function but needs attention; high risk indicates that some fault is present, and corresponding measures should be taken. Therefore, this paper adopts the comments set:

$$
M=\left\{M_{1}, M_{2}, M_{3}\right\}=\{\text { low risk, medium risk, high risk }\} .
$$

(2) Index Criteria. Data on bearing vibration, wheel set dimension, traction motor temperature, and thickness of brake shoes is collected at the operation field of a metro company. Based on results of data analysis, internal operating procedures, and related trade standards, the index standards are determined, as shown in Table 1.

Select four groups of evaluation sample data as shown in Table 2.

(3) Standard Domain and Extensional Domain. According to Table 1, the standard matter-element and extensional domain matrix are $R_{0}$ and $R_{P}$, respectively.

(4) Establishing the Correlation Function to Calculate the Degree of Correlation. Take sample 1 as an example; according to formulae (5) and (6), the degree of correlation matrix of the three level indexes under indices $B_{1}, B_{2}, B_{3}, B_{4}$, and $B_{5}$ is $K_{11}$, $K_{12}, K_{13}, K_{14}$, and $K_{15}$, respectively:

$$
\begin{aligned}
& K_{11}=\left[\begin{array}{ccc}
0.4114 & -0.5886 & -0.7120 \\
-0.0560 & 0.1470 & -0.2559 \\
0.2301 & -0.7699 & -0.8466
\end{array}\right], \\
& K_{12}=\left[\begin{array}{ccc}
0.1917 & -0.3485 & -0.4342 \\
0.1333 & -0.8667 & -0.9000 \\
0.4000 & -0.4000 & -0.5500 \\
0.1333 & -0.1333 & -0.3500
\end{array}\right], \\
& K_{13}=\left[\begin{array}{lll}
-0.1983 & 0.2333 & -0.0700
\end{array}\right], \\
& K_{14}=\left[\begin{array}{lll}
-0.4286 & -0.5714 & -0.6250
\end{array}\right], \\
& K_{15}=\left[\begin{array}{ccc}
0.4000 & -0.4000 & -0.6000 \\
0.3000 & -0.7000 & -0.8000 \\
-0.0200 & 0.0400 & -0.3288
\end{array}\right] .
\end{aligned}
$$

(5) Calculating the Weight of Indices. Use the degree of correlation matrix obtained above as input to the entropy weight method. 
TABLE 1: Index standards.

\begin{tabular}{|c|c|c|c|}
\hline Evaluation index & Low risk & Medium risk & High risk \\
\hline Effective value & {$[0.05,0.85]$} & $(0.085,0.1]$ & $(0.1,1)$ \\
\hline Peak factor & {$[2.5,3.2]$} & $(3.2,3.5]$ & $(3.5,30)$ \\
\hline Kurtosis & {$[2.5,3.5]$} & $(3.5,4]$ & $(4,30)$ \\
\hline Wheel diameter & $(780,840]$ & $(775,780]$ & {$[770,775]$} \\
\hline Wheel rim width & $(29,32]$ & $(28,29]$ & {$[26,28]$} \\
\hline Difference of coaxial wheel diameter & {$[0,1.5)$} & {$[1.5,2)$} & {$[2,10)$} \\
\hline Difference of co-bogie wheel diameter & {$[0,3)$} & {$[3,4)$} & {$[4,15)$} \\
\hline Traction motor temperature & {$[0,70)$} & {$[70,100)$} & {$[100,300)$} \\
\hline Brake shoe thickness & {$[20,55]$} & {$[15,20)$} & {$[0,15)$} \\
\hline Risk of elastic suspension device & {$[0,50)$} & {$[50,75)$} & {$[75,100)$} \\
\hline Risk of bogie frame & {$[0,50)$} & {$[50,75)$} & {$[75,100)$} \\
\hline Risk of ground device & {$[0,50)$} & {$[50,75)$} & {$[75,100)$} \\
\hline
\end{tabular}

TABLE 2: Four groups of sample data.

\begin{tabular}{|c|c|c|c|c|}
\hline Evaluation index & Sample 1 & Sample 2 & Sample 3 & Sample 4 \\
\hline Effective value & 0.0644 & 0.0741 & 0.0917 & 0.0566 \\
\hline Peak factor & 3.2241 & 3.3622 & 3.5621 & 3.3541 \\
\hline Kurtosis & 2.7301 & 2.9901 & 2.7782 & 3.0803 \\
\hline Wheel diameter & 791.5 & 786.2 & 782.3 & 782.9 \\
\hline Wheel rim width & 31.6 & 29.1 & 27.2 & 27.3 \\
\hline Difference of coaxial wheel diameter & 0.9 & 1.8 & 0.8 & 1.7 \\
\hline Difference of co-bogie wheel diameter & 2.6 & 3.9 & 3.85 & 1.7 \\
\hline Traction motor temperature & 93 & 61.6 & 92.8 & 52 \\
\hline Brake shoe thickness & 40 & 35 & 17 & 19 \\
\hline Risk of elastic suspension device & 30 & 24 & 26 & 23 \\
\hline Risk of bogie frame & 15 & 18 & 29 & 20 \\
\hline Risk of ground device & 51 & 40 & 55 & 47 \\
\hline
\end{tabular}

Use formulae (7)-(10) to calculate the weights of the three level indices:

$$
\begin{gathered}
\omega_{c_{1}}=\left(\omega_{c_{11}}, \omega_{c_{12}}, \omega_{c_{13}}\right)=(0.2557,0.5584,0.1859), \\
\omega_{c_{2}}=\left(\omega_{c_{21}}, \omega_{c_{22}}, \omega_{c_{23}}, \omega_{c_{24}}\right) \\
=(0.2162,0.1674,0.3344,0.2820), \\
\omega_{c_{5}}=\left(\omega_{c_{51}}, \omega_{c_{52}}, \omega_{c_{53}}\right)=(0.3554,0.2206,0.4240) .
\end{gathered}
$$

(6) Determination of Risk Level. Calculate the comprehensive correlation matrix $K_{1}$ of sample 1:

$$
K_{1}=\left[\begin{array}{l}
\omega_{B_{1}} \cdot K_{11} \\
\omega_{B_{2}} \cdot K_{12} \\
\omega_{B_{3}} \cdot K_{13} \\
\omega_{B_{4}} \cdot K_{14} \\
\omega_{B_{5}} \cdot K_{15}
\end{array}\right]=\left[\begin{array}{ccc}
0.1167 & -0.2115 & -0.4823 \\
0.2351 & -0.3918 & -0.5272 \\
-0.1983 & 0.2333 & -0.0700 \\
-0.4286 & -0.5714 & -0.6250 \\
0.2168 & -0.2796 & -0.5291
\end{array}\right] \text {, }
$$

while the criterion layer weights given by experts are

$$
\begin{aligned}
\omega_{B} & =\left(\omega_{B_{1}}, \omega_{B_{2}}, \omega_{B_{3}}, \omega_{B_{4}}, \omega_{B_{5}}\right) \\
& =(0.2360,0.2102,0.2404,0.1851,0.1283) .
\end{aligned}
$$

Using formula (11) to calculate the comprehensive correlative degree $K_{1}$ of sample 1 , the value is $(-0.0222,-0.2179$, $-0.4250)$. It can be known that sample 1 is at a low risk level. In the same way calculate the comprehensive correlative degree of sample 2, sample 3 , and sample 4 as $K_{2}=(0.0208$, $-0.0905,-0.6011), K_{3}=(0.1516,0.0373,-0.2570)$, and $K_{4}=(-0.1053,0.1534,-0.0918)$, respectively. The results of risk evaluation of sample 2 , sample 3 , and sample 4 are low risk, low risk, and medium risk, respectively. This is in high conformity with expert judgment.

\section{Conclusion}

Bogie system is a key system in the safe operation of a train. Accurate risk assessment can effectively prevent the occurrence of accidents, reduce part damage, and increase the utility of trains. This paper presents a risk assessment method of the bogie system based on extension theory and the entropy weight method. We consider the function and importance of various components of the bogie system and establish a risk evaluation method for the bogie system. Risk assessment results of different sample data of bogie system show that this method has good accuracy and can effectively determine the risk status of the bogie system. 
The method carries out evaluation for each index and can not only evaluate a single index but also evaluate multiple indices. But determination of the standard domain and extensional domain is needed during evaluation. At present there is no clear and effective method to define the threshold value. It is defined mainly by sample data training and commonly used work standard. Scientific and effective determination of grade threshold is one of the subsequent works that is needed. In addition, the revision and perfection of the index system of elements are needed in follow-up research.

\section{Conflict of Interests}

The authors declare that there is no conflict of interests regarding the publication of this paper.

\section{Acknowledgments}

The authors would like to express their thanks to the editor and anonymous reviewers for their help in revising the paper. This research is sponsored by the State Key Laboratory of Rail Traffic Control and Safety (no. RCS2011K013) of Beijing Jiaotong University.

\section{References}

[1] V. Belotti, F. Crenna, R. C. Michelini, and G. B. Rossi, "Wheelflat diagnostic tool via wavelet transform," Mechanical Systems and Signal Processing, vol. 20, no. 8, pp. 1953-1966, 2006.

[2] S. Jia and M. Dhanasekar, "Detection of rail wheel flats using wavelet approaches," Structural Health Monitoring, vol. 6, no. 2, pp. 121-131, 2007.

[3] J. Brizuela, A. Ibañez, and C. Fritsch, "NDE system for railway wheel inspection in a standard FPGA," Journal of Systems Architecture, vol. 56, no. 11, pp. 616-622, 2010.

[4] B. Chen, Z. Yan, and W. Chen, "Defect detection for wheelbearings with time-spectral kurtosis and entropy," Entropy, vol. 16, no. 1, pp. 607-626, 2014.

[5] D. Wang, Q. Miao, X. Fan, and H.-Z. Huang, "Rolling element bearing fault detection using an improved combination of Hilbert and wavelet transforms," Journal of Mechanical Science and Technology, vol. 23, no. 12, pp. 3292-3301, 2010.

[6] Y. Zhang, Y. Qin, Z. Xing, L. Jia, and X. Cheng, "Safety region estimation and state identification of rolling bearing based on statistical feature extraction," Shock and Vibration, vol. 20, no. 5, pp. 833-846, 2013.

[7] B. Suarez, J. M. Mera, M. L. Martinez, and J. A. Chover, "Assessment of the influence of the elastic properties of rail vehicle suspensions on safety, ride quality and track fatigue," Vehicle System Dynamics, vol. 51, no. 2, pp. 280-300, 2013.

[8] P. Li, R. Goodall, P. Weston, C. Seng Ling, C. Goodman, and C. Roberts, "Estimation of railway vehicle suspension parameters for condition monitoring," Control Engineering Practice, vol. 15, no. 1, pp. 43-55, 2007.

[9] T. X. Mei and X. J. Ding, "Condition monitoring of rail vehicle suspensions based on changes in system dynamic interactions," Vehicle System Dynamics, vol. 47, no. 9, pp. 1167-1181, 2009.

[10] M. An, Y. Chen, and C. J. Baker, "A fuzzy reasoning and fuzzyanalytical hierarchy process based approach to the process of railway risk information: a railway risk management system," Information Sciences, vol. 181, no. 18, pp. 3946-3966, 2011.

[11] S. Hong, Z. Zhou, E. Zio, and K. Hong, "Condition assessment for the performance degradation of bearing based on a combinatorial feature extraction method," Digital Signal Processing, vol. 27, no. 1, pp. 159-166, 2014.

[12] B. Sreejith, A. K. Verma, and A. Srividya, "Fault diagnosis of rolling element bearing using time-domain features and neural networks," in Proceedings of the IEEE Region 10 Colloquium and 3rd International Conference on Industrial and Information Systems (ICIIS '08), pp. 1-6, Kharagpur, India, December 2008.

[13] B. Samanta, K. R. Al-Balushi, and S. A. Al-Araimi, "Artificial neural networks and genetic algorithm for bearing fault detection," Soft Computing, vol. 10, no. 3, pp. 264-271, 2006.

[14] A. Mosallam, K. Medjaher, and N. Zerhouni, "Nonparametric time series modelling for industrial prognostics and health management," International Journal of Advanced Manufacturing Technology, vol. 69, no. 5-8, pp. 1685-1699, 2013.

[15] H. Xu, H. Yuan, L. Wang, W. Na, W. Xu, and Y. Li, "Modeling of metro wheel wear and optimization of the wheel re-profiling strategy based on Gaussian processes," Journal of Mechanical Engineering, vol. 46, no. 24, pp. 88-95, 2010 (Chinese).

[16] G. F. M. Dos Santos, L. A. S. Lopes, E. J. Kina, and J. Tunna, "The influence of wheel profile on the safety index," Proceedings of the Institution of Mechanical Engineers, Part F: Journal of Rail and Rapid Transit, vol. 224, no. 5, pp. 429-434, 2010.

[17] M. Ignesti, A. Innocenti, L. Marini, E. Meli, and A. Rindi, "Development of a wear model for the wheel profile optimisation on railway vehicles," Vehicle System Dynamics, vol. 51, no. 9, pp. 1363-1402, 2013.

[18] A. Bouscayrol, M. Pietrzak-David, P. Delarue, R. Peña-Eguiluz, P.-E. Vidal, and X. Kestelyn, "Weighted control of traction drives with parallel-connected AC machines," IEEE Transactions on Industrial Electronics, vol. 53, no. 6, pp. 1799-1806, 2006.

[19] C. Boccaletti, C. Bruzzese, O. Honorati, and E. Santini, "Rotor bars breakage in railway traction squirrel cage induction motors and diagnosis by MCSA technique part II: theoretical arrangements for fault-related current sidebands," in Proceedings of the 5th IEEE International Symposium on Diagnostics of Electric Machines, Power Electronics, and Drives (SDEMPED '05), pp. 16, Vienna, Austria, September 2005.

[20] M.-H. Wang and C.-Y. Ho, "Application of extension theory to PD pattern recognition in high-voltage current transformers," IEEE Transactions on Power Delivery, vol. 20, no. 3, pp. 19391946, 2005.

[21] X. Meng and L. Jia, "Train timetable stability evaluation based on analysis of interior and exterior factors information entropy," Applied Mathematics and Information Sciences, vol. 8, no. 3, pp. 1319-1325, 2014. 

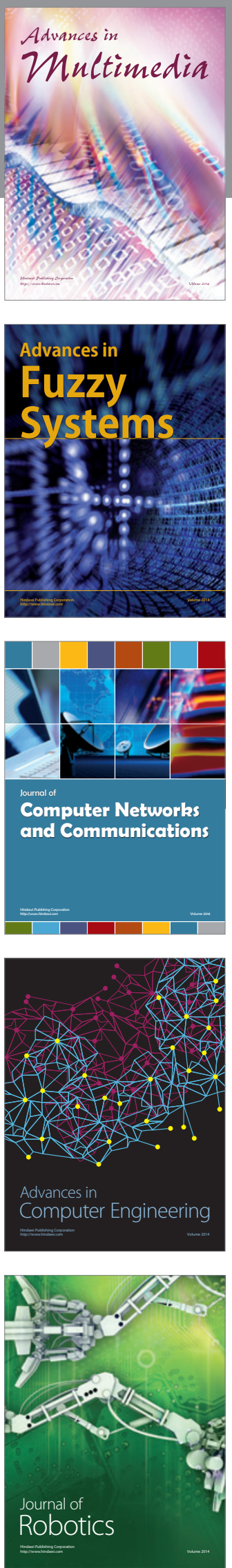

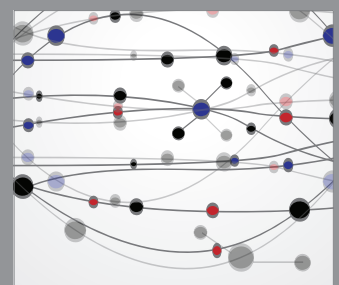

The Scientific World Journal
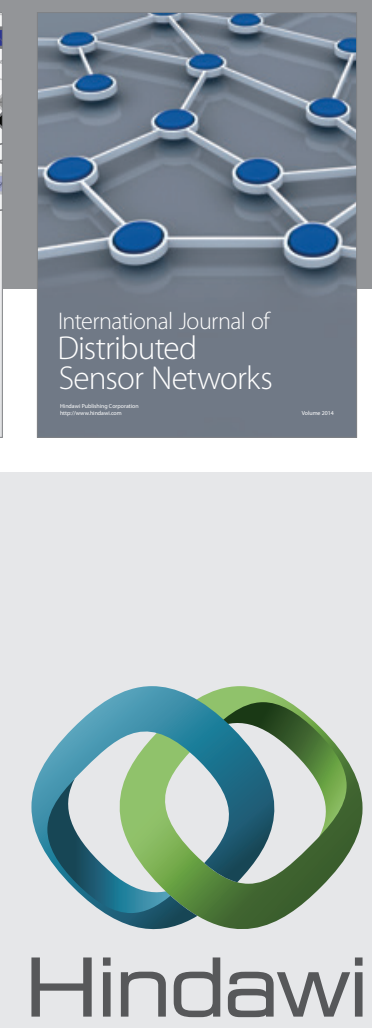

Submit your manuscripts at

http://www.hindawi.com
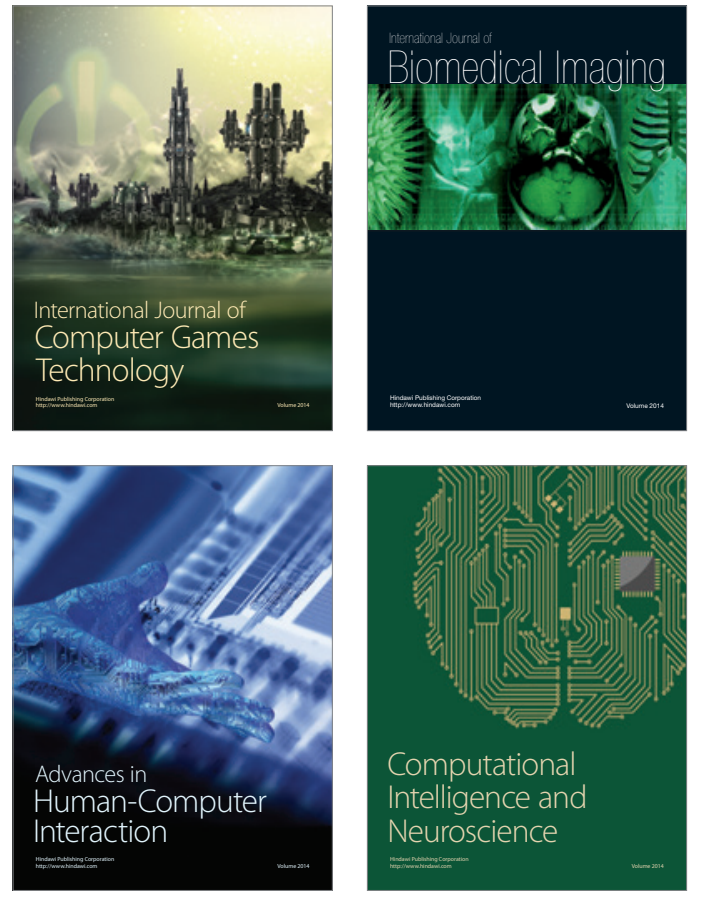
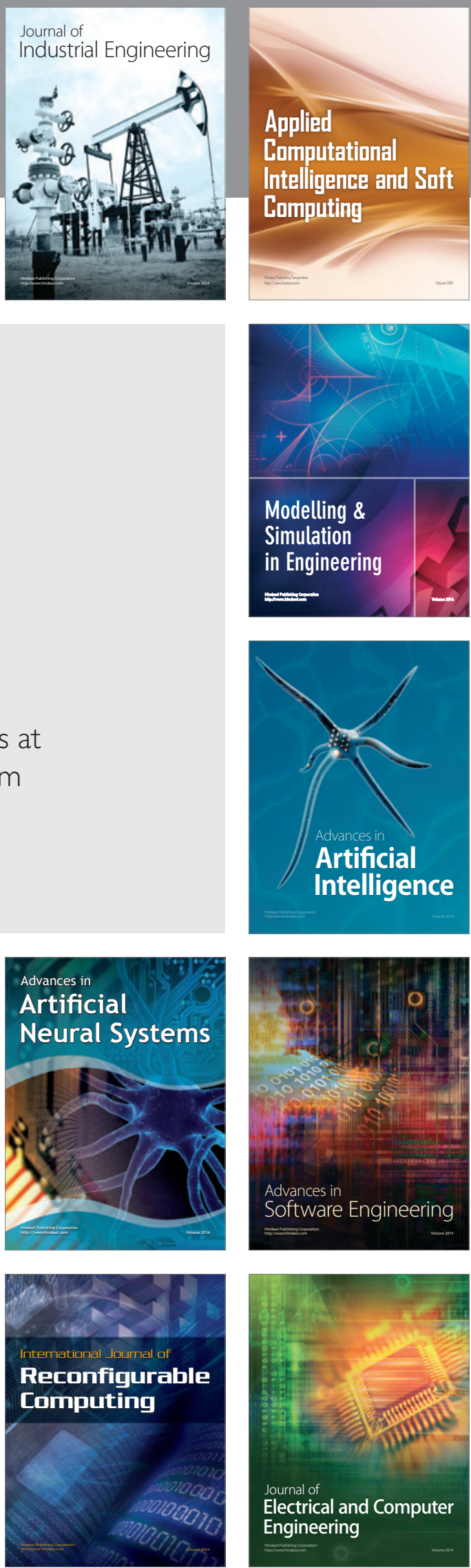